rev.relac.int.estrateg.segur.6(1):75-88,2011

\title{
EMPODERAMIENTO E HISTORIA, POR UNA FENOMENOLOGÍA DEL PODER INTERNACIONAL
}

Henry Cancelado*

\section{RESUMEN}

Dentro de la estructura del sistema internacional contemporáneo es cada vez más difícil dilucidar cuáles países son las potencias mundiales y en qué elementos basan su poder. La difusión actual de los elementos de poder tiene un doble efecto: por un lado hace que el sistema internacional parezca más anárquico, el sueño de los realistas, y que sea la fuerza de los estados la que organice el mundo y logre cierto equilibrio; por otro lado, tal anarquía y la explosión de unas economías incipientes, gracias a la crisis de las economías dominantes, hace que nuevos países sueñen con ser grandes potencias y cambien sus discursos. Así mismo, otros actores se empoderan y dictan, directa o indirectamente, reglas en el sistema internacional, reglas que empiezan a redefinir el poder en el mundo.

Palabras clave: Poder, capital, estrategias, sistema internacional.

* Politólogo y Magíster en Análisis de Problemas Contemporáneos; Docente Tiempo Completo de la Universidad Militar Nueva Granada. Correo: hcancelado@gmail.com 


\begin{abstract}
Within the framework of the current international system, it is increasingly difficult to clarify which countries are the world powers and on which elements they base their ruling. The current spread of the elements of power is twofold: on one hand, it makes the international system look more anarchic, the dream of the realists, and that the strength of the states be the one that organizes the world and achieves an equilibrium; but on the other hand, such anarchy and the outburst of emerging economies, thanks to the crisis of the dominant economies, make new countries dream of becoming great powers and so change their talk. Similarly, other actors grow empowered and dictate, directly or indirectly, rules in the international system, and those rules are beginning to redefine power in the world.
\end{abstract}

Key words: Power, capital, strategies, international system

\title{
RESUMO
}

Dentro da estrutura do sistema internacional contemporâneo é cada vez mais difícil elucidar quais países são potências mundiais e em que elementos se baseiam seu poder. A difusão atual dos elementos de poder tem um duplo efeito: por um lado faz com que o sistema internacional pareça mais anárquico, o sonho dos realistas, e que seja a força dos estados que organize o mundo e consiga certo equilíbrio; por outro lado, a anarquia e explosão de algumas economias incipientes, graças à crise das economias dominantes, faz com que novos países sonhem em ser grandes potências e mudem seus discursos. Do mesmo modo, outros atores se apoderam e determinam, direta ou indiretamente, as regras do sistema internacional, regras que começam a redefinir o poder no mundo.

Palavras chave: poder, capital, estratégias, sistema internacional.

\section{INTRODUCCIÓN}

Teniendo en cuenta los cambios acaecidos en la composición de los Estados modernos, a la salida de la Edad Media, se puede observar el cambio de una supra soberanía basada en el poder ultra terreno y terreno de la Iglesia Católica, al poder terreno de entidades humanas basadas en el manejo de un territorio, es decir, aparece un nuevo juego en la política mundial y es el juego de los Estados, los cuales se pueden entender como entidades vivas, basadas en una identidad que puede provenir de la raza o la historia, o de los componentes ideológicos identitarios que nutren los procesos de conformación de las naciones, las cuales son las bases de los Estados. 
El interés de un Estado se nutre de la suma de los intereses particulares que a la larga lo van a llevar a actuar en la arena internacional. Sin lugar a duda, el Realismo acierta cuando afirma que lo que le interesa a un Estado es su supervivencia, lo que quede más allá de eso son los aspectos tácticos y estratégicos de la supervivencia de un Estado. Aspectos que se redefinen dependiendo de la coyuntura del sistema internacional.

La sucesión de poderes en el sistema internacional a lo largo de su historia, España, Gran Bretaña, Estados Unidos, por mencionar algunos, muestra que el poder se materializa en entidades concretas, que incluso antes de hablar de un sistema internacional clásico ya existía poder mundial que se transfería dentro de las diferentes unidades que existían en el mundo, imperios, tribus, iglesia, por mencionar los más importantes. Sin embargo, cuando se alude a un estudio del sistema internacional contemporáneo, se puede observar la lucha de unos Estados que se niegan a dejar de ser los detentadores de un poder a nivel internacional, detentadores exclusivos del destino y la suerte del sistema internacional, lastimosamente para ellos, su escenario de acción cada vez se ve menos reducido a ciertos escenarios del sistema internacional, importantes o no, eso es otro debate. Con excesivo cuidado de no caer en una especie de marxismo que sentencia al Estado a servirle a intereses privados y extraños al interés común, es necesario analizar si de verdad el poder internacional se queda en los escenarios territoriales y aparentemente soberanos que llamamos estados, o si aparecen en nuevas entidades que no se valoran dentro de la modernidad de las relaciones internacionales por considerarlas débiles frente a los Estados.

Dentro del "metajuego de la política mundial" (Beck. 2004) hay enfrentamiento de las formas antiguas y nuevas de hacer la política. Esto se refiere en cuanto a la multiplicidad de procesos y al escenario entreverado de actores y alianzas, de intereses y de estrategias. Si se entiende el poder mundial como un ejercicio que se reconoce a través de sus efectos, debido a que es un ejercicio que genera procesos y que conlleva consecuencias, (Cancelado. 2010) que crea instituciones y que las destruye, cambia paradigmas y obliga, dentro de las estrategias por su consecución, a nuevas tácticas.

El objetivo de este artículo es analizar las formas en que el poder se materializa en el sistema internacional. Para tal fin es necesario partir del supuesto de que el poder siempre se ha concretizado en diferentes entidades a nivel mundial, pero eso no significa que haya una sola entidad que lo personifica de manera exclusiva, sino que el sistema mundial tiene elementos fuertes de poder que son asumidos por entidades, o instituciones y elementos subsidiarios del mismo poder, que en últimas generan redes de poder, de resistencias y de hegemonías que crean la apariencia de orden. En la remoción de estas redes, en la aparición de estos nuevos elementos, aparecen los cambios y las crisis globales. 


\section{EL PODER METAFÍSICO}

"No tendrías ningún poder sobre mí, si no se te hubiera dado de arriba"

(Juan 19:11)

Afirmar que dentro del mundo no existe la posibilidad de un vacío de poder es temerario, pero a la vez real, en el sentido en que el poder, como inmanencia que mueve la historia, es en sí mismo una fuerza que se materializa donde quiere y como quiere dependiendo el contexto; el poder depende de espacio y tiempo para actuar de una cierta manera.

En la antigüedad, el poder se estableció básicamente dentro de unidades políticas, de corte tribal, que se fueron sumando hasta crear las formas imperiales que dominaron el mundo hasta el 500 d.C. aproximadamente, época en la cual algunas naciones fusionadas provenían de tribus que lucharon a lo largo de 5.000 años por mantener alguna autonomía o determinación política, económica y religiosa (Osborne, 2007).

En el caso occidental se tiene la resistencia de las ciudades griegas ante la expansión del Imperio Persa a lo largo de las Guerras Médicas en el siglo V a.C. Imperio conformado a su vez por multiplicidades de tribus y ciudades - estado, a su vez provenientes de pueblos dorios, jonios y etruscos del mediterráneo (Osborne, 2007), En las Guerras Médicas, la resistencia griega representa un contrapoder a la entidad dominante, en este caso el Imperio Persa. Igualmente, con la expansión del Imperio Macedónico, en el siglo III a.C., el mundo observa un cambio en la tendencia dominante del poder. Al mismo tiempo que nace una nueva entidad con el favor del poder, la entidad anteriormente dominante empieza a representar la resistencia, el contrapoder. En este punto es necesario entender que una de las características del poder es su amoralidad, la moralidad del poder la ponen las entidades que lo personifican, independientemente de si son las dominantes o no. Se establece una dialéctica entre las entidades por ganar el favor del poder; pero a nivel global, el poder se mueve libremente y genera cambios o continuidades dependiendo de su conveniencia a nivel mundial.

Con la aparición del poder mediterráneo empiezan a florecer diferentes asentamientos como Roma, Cartago y algunas colonias griegas en la actual España. El crecimiento de estos asentamientos, la expansión de estas ciudades por el mediterráneo y la necesidad de supervivencia y de comercio lleva a las Guerras Púnicas. La exacerbación del poder, concentrado en la región mediterránea, materializado en pueblos que crecen comercial, militar y políticamente hacen que haya un desbordamiento de tal concentración, es decir, demasiado poder entre muchos que tienen un interés, una moral, una forma de entenderse a sí mismo y a los demás y una forma de comprender su soberanía y de manejar su poder. Cuando estas visiones del poder propio y del poder de los demás se enfrentan, aparece el conflicto como forma de dirimir tal disputa; alguna entidad triunfa, otra pierde, pero el poder se mantiene, solamente que en nuevas formas y nuevas estructuras militares, económicas e ideológicas. 
Las Guerras Púnicas se resuelven a favor de Roma, que se fortalece e inicia su proceso de expansión hasta su declive en el 476 d.C. y pasa por diferentes etapas, pero va a llegar a Imperio, con la anexión de territorios en Europa, Medio Oriente y el Norte de África. De igual manera, como el Imperio Persa había logrado homogenizar y dominar tribus en antaño, el Imperio Romano logra hacer lo mismo en estas regiones, logra incluso el dominio de otros imperios como el egipcio. Una de las características de los macedonios y de los romanos es que respetaron algunos credos y cultos locales, el elemento religioso era clave en el poder internacional. Este componente religioso va a ser determinante en la caída de los romanos.

Dentro del imperio va a aparecer, con gran fuerza, un poder totalmente inmanente y supramundano, de alguna manera sobrenatural, que va a destruir las visiones del poder romano que tenían los ciudadanos imperiales y los dominados por estos. El cristianismo primitivo logró subvertir los procesos del poder imperial y llevar al poder al terreno metafísico con una eficiencia que antes no se había logrado. Los emperadores antiguos, hasta el emperador de China, se asumían hijos o descendientes de los dioses. El punto central con el cristianismo es que destruyó la entronización imperial del poder divino y lo puso a la altura de los siervos y de los lacayos del imperio; como consecuencia, hay una subversión del orden terrenal desde lo supra-terrenal; la forma natural y material del poder se traslada a un orden mayor y superior al César. Frases como: Dad al César lo que es del César y a Dios lo que es de Dios (Mt. 22, 15-21) o, No tendrías ningún poder sobre mí si no se te hubiera dado de arriba (Juan 19:11), que aparentemente son desprevenidas, tienen dentro de la teología una intención clara de subversión del poder natural y terrenal; colocan la figura del héroe cristiano por encima del poder del imperio. Esto hizo que la forma de seguimiento cristiano en los primeros siglos de esta era fuera la emulación del martirio y del desafío al poder romano. Roma nunca lo entendió a plenitud, por eso es que intentó perseguir y asesinar a los cristianos, el pequeño problema es que los cristianos, doctrinalmente hablando, no mueren. El desafío al poder del imperio radicó en: no importa que asesine a los cristianos, la muerte es triunfo y el asesinato, como única estrategia, demuestra su fracaso frente a nuestro Dios; por consiguiente, somos más poderosos. De ahí que, después de siglos de persecución, el orden romano fue subvertido y las bases sociales del imperio, que radicaban en el respeto y obediencia a la figura del César, cambiaran y el poder de Dios se instaurara en el orden terrenal. Algunos líderes doctrinales como Agustín de Hipona, Evagrio Ponto e Ignacio de Antioquía, por nombrar algunos, se enfrascaron en nuevas luchas, luchas contra las llamadas herejías, contra los arrianos y contra los maniqueos; en nuevos escenarios, los salones de disputa y los púlpitos, el poder religioso de la cristiandad antigua había destruido al imperio. Roma nunca lo entendió, Roma había desaparecido.

Nuevos emperadores como Carlo Magno ya no conquistaban, ni siquiera en pos de una nación, de un territorio, sino de un nuevo tipo de emperador, aquel que conectaba dos realidades: la natural y la sobrenatural, una nueva cabeza había reemplazado al César: el Papa romano, quien tenía todo el poder en esta tierra y el poder en la siguiente, de acuerdo con la doctrina cristiana entronizada en su momento. Poder que va a ser disputado y que va a dar espacio a las guerras 
de los siguientes 1.400 años, cuando el poder del Imperio regresa por lo suyo, cuando Napoleón I le arrebató la potestad de la coronación al Papa Pío VII en 1804. Nunca más un papa determinaría los destinos de imperios, Estados o entidades terrenales, de la manera tan definitiva como hasta ese momento.

Pero entre el siglo IV d.C. y 1804, el poder religioso tuvo una serie de guerras por mantenerse y tuvo una serie de contrapoderes que le intentaron arrebatar la categoría de entidad dominante (Patiño. 2005: 24); sin embargo, no van a ser solamente las guerras sino una nueva entidad la que logrará crear nuevas instituciones en el mundo: el racionalismo científico y la modernidad cartesiana y anticlerical.

\section{EL PODER IDEOLÓGICO Y RACIONAL}

"Voy a dudar de todo, de lo único que no voy a dudar es que estoy dudando".

René Descartes

Lutero nunca imaginó que su deseo de regresar a las fuentes del cristianismo, después de 1.000 años de dominio de la Iglesia Católica, y de casi 1.300 años de que Roma como ciudad fuera el centro del mundo, le abriera paso a formas totalmente contrarias a su deseo inicial (Weber, 2003). En la explosión ideológica y religiosa del siglo XVI, es decir, en la lucha dogmática de esos años, se abrió el escenario perfecto para nuevas ideas, no religiosas, pero tan subversivas como los cristianos de los primeros siglos. Nuevamente una dialéctica aparece en este estadio histórico. Después de 1.000 años desde que el cristianismo destruyera al Imperio Romano, luchara contra las herejías y buscara su expansión y la defensa de su orden feudal europeo en las Cruzadas, entre los años 1090 y 1300, era obvio que los procesos de su poder y la forma de entender su poder en el mundo generara resistencias cada vez mayores y más fuertes. Esta vez vino desde el mismo corazón del cristianismo. La Roma cristiana expulsó las amenazas religiosas en España, logró el dominio de tierra santa, luego lo perdió, sometió a los judíos, a los gitanos y estableció un orden político, militar y económico que servía a sus intereses religiosos. Alineó a Europa y a sus nuevas colonias en un orden desde el poder metafísico en el que se basaba, el dios despreciado por los romanos antiguos ahora era reconocido en todo el mundo. A pesar de este inmenso dominio, corrió con la misma suerte de persas y romanos.

El golpe no vino desde fuerzas exteriores a sí misma, sino que apuntaron desde lo más fuerte que tenía: la doctrina a su reforma, reforma que no pudo controlar nuevas entidades que encontraron en este nuevo discurso la mejor forma de resistir y de destronar al poder dominante (Osborne, 2007: 254). Si bien la reforma protestante apuntó al cambio radical de las formas cristianas del 
poder, no entendió que las resistencias venían, no solamente desde ese movimiento, sino desde muchos otros que no necesariamente tenían formas religiosas, pero sí dogmáticas y subversivas como el humanismo de finales del siglo XV. Pero tampoco entendió que el poder doctrinal de la Iglesia Católica estaba acompañado de un gran poder político, económico y militar, producto de 1.000 años de dominio, de relaciones con la nobleza, de guerras y de comercio. Como resultado de la ignorancia contextual de Lutero y de su posterior y radical emulador, Calvino, todo el cristianismo perdió el poder que tenía y quedó a merced de su peor enemigo, el racionalismo del siglo XVII.

Lutero debilitó el poder doctrinal cristiano y las guerras de religión debilitaron el poder terrenal del mismo. Por consiguiente, Westfalia no es más sino el resultado, en 1648, de un festín del poder mundial, concentrado durante mucho tiempo y ahora repartido entre varios nuevos poderosos, el más importante: el Estado.

\section{LAS TRES REVOLUCIONES BURGUESAS Y EL SIGLO XX. EL PODER PRAGMÁTICO}

El siglo XVIII cerró con éxito para el proyecto liberal racionalista. Si bien el experimento republicano Inglés del siglo anterior fue un fracaso que terminó con la restauración de la monarquía, los experimentos derivados de las tres revoluciones de finales de siglo XVIII y de comienzos del siglo XIX dejaron marcas indelebles en el sistema internacional.

En primer lugar la Revolución Americana, 1776, se erigió como el perfecto ejemplo del éxito de una nación que se consideraba a sí misma soberana frente al poder real de Jorge III, y redefinía al Estado dentro de sus escenarios internos de poder, es decir, al cambiar el concepto de soberanía, cambió la situación del poder. Los Estados Unidos lograron ser la primera nación que pudo combinar los elementos filosóficos del liberalismo político, encarnada básicamente en John Locke, y que planteaba dos temas centrales para la filosofía política: el problema de las teocracias y el problema de los absolutismos (Locke, 1990; Spinoza, 1986). En este sentido, la filosofía ya había avanzado lo suficiente desde el escepticismo cartesiano, pasando por la rebelión anticlerical y antimonárquica de Spinoza, estableciendo una nueva forma de pensar la política y la teología. Al sumar estas iniciativas intelectuales con procesos históricos tales como el continuo ascenso de la burguesía y, como se dijo antes, la destrucción de la forma institucional ideológico-política de la Iglesia Católica, se tiene el campo de cultivo de una forma ideológica que los revolucionarios estadounidenses van a entender muy bien. La entienden tan bien, que cargan consigo la misma contradicción de Lutero: por un lado propugnaban por un Estado con valores divinos, tal y como lo muestra su Acta de Independencia; pero por otro lado abrieron todo el escenario para la privatización de la religión, el desplazamiento de la teocracia y la aparición de una democracia secular. 
En segundo lugar, La Revolución Francesa, 1789, estableció los criterios universales de la igualdad y de los derechos humanos (Gandini, 1998). Preceptos que dieron la posibilidad a que la burguesía, otrora segregada en torno a un sistema político y económico, empezara a ser incluida en los procesos de participación política y, por supuesto, legitima su concentración de poder en torno al concepto de ciudadanía, en el cual, si bien se establece un principio de soberanía y se entroniza un nuevo actor de poder, en este caso, resistente al poder hegemónico del Estado absolutista, da como resultado un nuevo tipo de Estado, con procesos más amplios de participación, basado inicialmente en los reducidos derechos del hombre, los cuales darán pie a toda una serie de derechos colectivos, individuales, específicos y genéricos del ciudadano y se establece un principio liberal de limitación del poder del Estado frente al poder del individuo.

Tanto en Estados Unidos como en Francia, el doble proceso de creación de ciudadanía con ampliación de los derechos y el de reafirmación de la individualidad hace que el proceso ideológico previo, es decir, el de la identificación institucional con la Iglesia y con el monarca, se vea superado. La combinación de derechos con libertad individual da como resultado una forma de poder que establece la soberanía desde los procesos sociales con una forma ideológica racionalista y colectiva, basada en la forma instrumental de la razón, alejándose de los procesos ideológicos de la razón pura. De tal manera la racionalidad se entroniza como el nuevo articulador de las relaciones sociales, en consecuencia, deriva en el instrumentalismo pragmático.

Por último, la Revolución Industrial inglesa permitió el surgimiento de un modelo económico moderno. Las formas patrimoniales, basadas exclusivamente en la tenencia de la tierra, se transforman en el sentido en que nuevos tipos de relaciones comerciales $y$, sobre todo, nuevas formas de entender un sistema económico mundial empiezan a tomar relevancia en el sistema internacional. El doble proceso económico que se lleva desde finales del siglo XVIII ayudará a sustentar procesos políticos e ideológicos expuestos anteriormente, y toda la transformación de los procesos de producción nacional, en tanto que el desarrollo propio de la Revolución Industrial aceleró la producción industrial y creó un excedente que aumentó la capacidad de acumulación y la posibilidad de mayor flujo comercial.

Los cambios que se produjeron durante este período (1760-1850), de hecho, se produjeron de forma gradual. En realidad, habían comenzado hacía más de dos siglos. A finales del siglo XVIII y principios del siglo XIX llegaron a buen término las ideas y los descubrimientos de todos los pensadores de esa época, por ejemplo, Galileo, Bacon, Descartes y otros.

El sistema de la fábrica había cambiado la manera en que el trabajo se llevó a cabo. Poco a poco, los trabajadores comenzaron a darse cuenta de la fuerza que podía tener si se tratara de una fuerza unificada. Fue una batalla larga para que los trabajadores pudieran organizarse en sindicatos reconocidos oficialmente. De tal manera las ideas nuevas frente a la capacidad de la clase obrera tuvieron gran acogida y dio la idea de que una especie de clase mundial pudiera 
organizarse para detener una amenaza, en este caso no de un Estado ni de un tirano, sino de un sistema. El Estado era simplemente visto como su arma más poderosa (Marx).

\title{
4. UN MUNDO INCIERTO, O EL MUNDO DEL CAPITAL. EL PODER ABSOLUTO
}

\author{
"El capitalismo sólo triunfa cuando se identifica con el Estado, cuando es el Estado." \\ Fernand Braudel
}

El problema del poder actual es cuando se desterritorializa; en ese sentido, el poder escapa a conceptos de soberanía y de control. Pasa a ser un poder inmanente que es totalmente fiel a sí mismo y que no tiene entidad o identidad como tal. Con ese poder etéreo, y con los cambios acaecidos, pareciera que la economía tomara una relevancia que antes no era tan clara, en la cual el Estado pasa a proteger intereses económicos y quebranta elementos de su naturaleza clásica para pasar a una forma de poder instrumental.

Existe entonces una sucesión de paradigmas económicos desde la Edad Media en tres momentos distintos, cada uno definido por el sector dominante de la economía: un primer paradigma en el que la agricultura y la extracción de materias primas dominó la economía, un segundo en que la industria y la fabricación de bienes durables ocuparon la posición de privilegio, y un tercer paradigma y actual en la que la prestación de servicios y la manipulación de la información están en el corazón de la producción económica y, por supuesto, en el centro del debate político también. La posición dominante ha pasado por lo tanto de primaria y secundaria a la producción terciaria. La modernización involucra el pasaje del primer paradigma al segundo, de la dominación de la agricultura a la de la industria. Modernización significa la industrialización, pero ¿cómo llamar a lo nuevo que se tiene hoy en día? Hablar de una posmodernización es afirmar que procesos económicos y políticos propios de la modernidad ya se han superado y no es así.

Así como los procesos de industrialización transformaron la agricultura y la hicieron más productiva, dándole fuerza económica y política al Estado, también la revolución de la información transformará la industria redefiniendo y rejuveneciendo los procesos de fabricación. En efecto, como se transforman las industrias, la división entre la industria y los servicios es cada vez más imprecisa y la definición de políticas públicas para el sector productivo también. Al igual que en el proceso de modernización toda la producción tendió a industrializarse, así también actualmente toda la producción tiende hacia la producción de servicios y al manejo de la información. Los Estados entran en una pugna con otros actores por el manejo de una información desterritorializada, pero altamente importante para el control político y el desarrollo nacional.

No todos los países, por supuesto, incluso entre los países capitalistas dominantes, han asumido el mundo contemporáneo de la misma manera. Manuel Castells (2005) ya advertía sobre los 
procesos actuales en el aumento del empleo en los servicios, las relaciones entre los diferentes servicios y manufactura. El primer camino tiende hacia un modelo de economía de servicios y está dirigido por los Estados Unidos, el Reino Unido y Canadá. Este modelo implica una disminución de identificación de puestos de trabajo industrial y un correspondiente aumento rápido en empleos del sector servicios, en particular, los servicios financieros que manejan el capital llegando a dominar los sectores de servicios. En el segundo modelo, el modelo de información industrial, caracterizado por Japón y Alemania, la disminución del empleo industrial más lentamente lo hace en el primer modelo y, más importante, el proceso de informatización está estrechamente integrado y sirve para reforzar la fuerza de existentes de producción industrial. Servicios relacionados directamente con la producción industrial, por lo tanto, siguen siendo más importantes en este modelo en relación a otros servicios. Los dos modelos representan dos estrategias para gestionar y obtener una ventaja en la transición económica, pero debe quedar claro que ambos se mueven con decisión en la dirección de la informatización de la economía y la creciente importancia de los flujos y redes productivas.

Aunque los países subordinados y regiones del mundo no son capaces de aplicar estas estrategias, hoy, no obstante, se imponen cambios irreversibles en ellos. El hecho de que la informatización y el cambio hacia los servicios se han realizado hasta ahora principalmente en los países capitalistas dominantes y no en otra parte, no debe llevarnos de nuevo a la comprensión de la actual situación económica mundial en términos de etapas lineales de desarrollo. Es cierto que a medida que la producción industrial ha disminuido en los países dominantes, ha sido efectivamente exportada a países subordinados, de los Estados Unidos y Japón, por ejemplo, a México y Malasia. Tales desplazamientos geográficos podrían llevar a algunos a creer que hay una nueva organización global mediante la cual los países dominantes son economías de servicios de información y sus subordinados primeras economías industriales y agrícolas.

Es cada vez más evidente desde la perspectiva de las regiones subordinadas que la modernización ya no es la clave para el progreso económico, la competencia y la inserción internacional. Las regiones más subordinadas, tales como áreas de África subsahariana, están excluidas de los flujos de capital y nuevas tecnologías y, por lo tanto, se encuentran al borde de la inanición. La competencia por las posiciones de nivel medio en la jerarquía global no es llevada a cabo a través de la industrialización, sino a través de la informatización de la producción.

En este sentido, una nueva forma de poder actual, enfocado a los sistemas del capital y a sus procesos de reproducción y difusión, daría la sensación de que las relaciones internacionales actuales se mueven por intereses que no necesariamente son el temor a la guerra o al poder militar del enemigo. Las invasiones de Estados Unidos en Medio Oriente parecen ser unas combinaciones convenientes de un poder militar que busca derrotar a sus enemigos, pero que termina dejándole los territorios conquistados al capital internacional, a las empresas globales. Dentro de esta lógica, mientras existan este tipo de intereses, siempre habrá conflicto, pero cada vez más tecnológico, más táctico, más preciso. 
El paso hacia una nueva economía internacional implica necesariamente un cambio en la calidad y la naturaleza del trabajo. Esta es la consecuencia más inmediata sociológica y antropológica del pasaje de paradigmas económicos. Hoy en día la información y la comunicación han llegado a desempeñar un papel fundamental en los procesos de producción.

La revolución informática y la comunicación de la producción han transformado las prácticas de trabajo de parto de tal manera que todos ellos tienden hacia el modelo de tecnologías de la información y la comunicación interactiva y máquinas cibernéticas, convertido en una nueva prótesis integrada en nuestros cuerpos y mentes y una lente con la qué redefinirlos ellos mismos. La antropología del ciberespacio es en realidad un reconocimiento de la nueva condición humana.

Aparece un tipo de trabajo inmaterial implicado en equipo y comunicación, que reclama el valor más alto y, por lo tanto, se identifica como la clave para la competencia en la nueva economía global (Reich, 1993). El crecimiento de estos puestos de trabajo, basados ??en el conocimiento de la manipulación simbólica creativa, implica un crecimiento correspondiente de bajo valor y el empleo de baja cualificación de la manipulación de símbolos de rutina, tales como la entrada de datos y procesamiento de textos. Aquí comienza a surgir una división fundamental del trabajo en el ámbito de la producción inmaterial.

Hay que señalar que una de las consecuencias de la informatización de la producción y la aparición del trabajo inmaterial ha sido una homogeneización real de los procesos de trabajo de parto. Desde la perspectiva de Marx, en el siglo XIX, las prácticas concretas de diferentes actividades laborales eran radicalmente heterogéneas: corte y confección y tejido involucrando acciones concretas inconmensurables. Solo cuando se abstrae de sus prácticas concretas podían diferentes actividades laborales se reunió y visto de una manera homogénea, ya no es tan largo y tejer, pero como el gasto de fuerza de trabajo humana tomada en general, como trabajo abstracto. Con la informatización de la producción hoy, sin embargo, la heterogeneidad del trabajo concreto ha tendido a reducirse, y el trabajador está cada vez más lejos del objeto de su trabajo. El trabajo de sastrería computarizada y el trabajo de tejer computarizado pueden implicar exactamente las mismas prácticas concretas, es decir, la manipulación de los símbolos y la información. Las herramientas, por supuesto, siempre han abstraído fuerza de trabajo del objeto de trabajo, hasta cierto punto. A través de la informatización de la producción, entonces, el trabajo tiende hacia la posición de trabajo abstracto.

En este proceso de abstracción y de cambio en el sistema productivo internacional, el poder se materializa constantemente en los movimientos de resistencias, así como en los movimientos hegemonizantes. Adicionalmente, este proceso de flujos internacionales no permite que exista una entidad territorial lo suficientemente poderosa como para detener lo intangible. Solamente se puede detener desde la crisis de los sistemas productivos que hoy en día son globales. 
Si bien, anteriormente el Estado podía imponerse como un espacio casi que exclusivo de dominio y manejo de todos los procesos sociales, tanto nacionales como internacionales, el capitalismo ha ido produciendo el espacio abstracto que contiene el mundo de la mercancía, su lógica y sus estrategias a escala mundial, al mismo tiempo que el poder del dinero y del Estado político. La conexión entre el espacio y la expansión del capitalismo, sustituyendo el espacio físico por un espacio ideológico y luego por uno virtual, ha llevado a repensar el sistema internacional, sus instituciones y los alcances de las mismas.

\subsection{El nuevo poder}

La economía mundial está en relación con el Estado como una especie de meta-poder que puede cambiar las reglas nacionales e internacionales. La economía se ha escapado de la jaula de conflicto de poder territorial, se ha organizado a nivel nacional y ha adquirido nuevos movimientos de energía en el espacio digital. Es exactamente lo contrario de la teoría clásica del poder: la amenaza ya no de una invasión, sino de la no-invasión de los inversores. Solo hay una cosa peor que ser invadido por las grandes multinacionales: no ser invadido por las multinacionales.

Este tipo de autoridad no está vinculado a la ejecución de órdenes, sino a la posibilidad de ir a otro sitio o a otros países a tomar mejores inversiones. Se presenta la amenaza de no hacer algo, es decir, de no invertir en este país. El nuevo poder económico global de las grandes empresas no se basa en la violencia como la última razón y esto hace que sea mucho más móvil; el poder no está atado a ningún lugar específico y, por tanto, puede eliminarse a nivel mundial.

Si bien el poder de los estados (de acuerdo con la lógica nacional) crece a través de la conquista territorial, el poder de los actores de la economía mundial crece precisamente en la medida en que se conviertan en factores extraterritoriales. El poder del Estado se expresa en su relación con otros estados mediante la amenaza militar o de conquista, pero el nuevo tipo de poder se desterritorializa a través del comercio transnacional y la actividad en el espacio digital. Esta concepción invierte la lógica de la concepción tradicional del poder, la violencia y la autoridad. El poder desterritorializado no necesita ser políticamente obtenido ni legitimado. Su aplicación evita las instituciones de la democracia desarrollada, como los parlamentos y los tribunales; este meta-poder no es ni ilegal ni legítimo, pero cambia las reglas de los sistemas nacionales e internacionales.

El poder de no invertir o invertir existe en todas partes. Es la regla de nadie. Nadie está a cargo, nadie lo empezó, nadie lo puede detener. Cuanto más el discurso de la globalización domina todos los ámbitos de la vida, las estrategias de capital más poderosas son. Pero esto no significa que los gerentes están gobernando el mundo. Es importante destacar que el meta-poder no depende de ellos como persiguiendo una agenda política. Más bien, pasan a hacer "política" 
como un efecto secundario. Su participación no es ni política ni apolítica. Es simplemente un elemento subsidiario del poder.

\section{Aporía final}

Hasta ahora las reglas del juego en la política mundial han sido violentas e imperialistas. Se ha pasado desde las formas guerreras y supranaturales, hasta las formas racionalistas y soberanas. Hoy en día no es más pacífico, pero tiene nuevas estrategias que hacen que los costos humanos se transfieran del campo de batalla a los costos sociales de la producción. El poder del capitalismo global se deriva de su potencial, es altamente invasivo y, dependiendo el interés, utiliza los recursos del Estado que sigue teniendo el monopolio de la violencia. Por supuesto, el capital global tiene que estar localizado en alguna parte, pero lo está en ninguna a la vez, es local y global, al mismo tiempo.

Este nuevo tipo de poder puede amenazar el orden anterior del sistema internacional, pero puede correr dos tipos de suerte: o establecerse a lo largo del tiempo y paulatinamente, como en la Revolución Industrial, o dar un golpe violento que intente subvertir el orden actual, como en la Revolución Francesa. Parece ser que se dirige más al estilo inglés, silencioso, continuo y efectivo, con crisis, pero no derrotado; es un nuevo poder que redefine unas nuevas relaciones internacionales desde la economía política internacional y el comercio global. Al estilo francés es inviable, fue un movimiento explosivo, pero débil, que perdió todo su poder. Al final los reyes regresaron.

\section{BIBLIOGRAFÍA}

- Amin, S. (2004). Geopolítica del imperialismo contemporáneo. En Clacso (Eds.). Nueva Hegemonía Mundial. $(73-110)$. Buenos Aires: Clacso.

- $\quad$ Beck, U. (2004). Poder y Contrapoder en la Era Global. Barcelona: Paidós.

- Beck, U. (1998). ¿Qué es la globalización?, Barcelona: Paidós.

- Berger, M. \& Huntington S. (2002) Globalizaciones múltiples. Barcelona: Paidós.

- $\quad$ Braudel, F. (1982). Civilization and Capitalism 15th - 18th century. New York: Harper and Row.

- Braudel, F. (1984). Civilización material, economía y capitalismo, siglos XV-XVIII. Alianza. Madrid.

- Cancelado, H. (2006). Las Redes de Poder en el Sistema Internacional. Análisis desde El Caso Colombiano. Revista de la Facultad de Relaciones Internacionales, Estrategia y Seguridad, Vol 1, № 2.

- Cancelado, H. (2010). El final del concierto europeo: confluencia de política y economía. Revista de la Facultad de Relaciones Internacionales, Estrategia y Seguridad, Vol. 4, №1. 
- Castells, M. (1999) La era de la información: economía, sociedad y cultura., Análisis Político, № 37.

- Castells, M. (2005). La era de la información economía, sociedad y cultura. Madrid: Alianza Editorial.

- Chomsky, N. (2002). La estructura emergente del orden mundial. Bogotá: Universidad Nacional de Colombia.

- Cox, O. (1972). El capitalismo como sistema. Caracas: Editorial Fundamentos.

- $\quad$ Fazio, H. (2002). La Globalización en su Historia. Bogotá: Universidad Nacional de Colombia.

- Foucault, M. (1980). Microfísica del poder. Madrid: Las Ediciones de la Piqueta.

- Foucault, M. (2006). Seguridad, territorio y población. Buenos Aires: Fondo de Cultura Económica.

- $\quad$ Friedman, J. (2001). Identidad cultural y proceso global. Buenos Aires: Amorrotu Editores.

- García, N. (1990). Culturas híbridas, estrategias para entrar y salir de la modernidad. México: Grijalbo.

- Grimsom, A. (2000). Interculturalidad y comunicación. Bogotá: Editorial Norma.

- Held, D. (2002). Transformaciones globales. México: Oxford.

- Hobsbawm, E. (1999). Historia del Siglo XX. Buenos Aires: Editorial Crítica.

- Kennedy, P. (1992). Auge y caída de las grandes potencias. Barcelona: Plaza y Janés.

- $\quad$ Keohane, R. (1988). Poder e Interdependencia. Buenos Aires: Editorial GEL.

- Locke, J. (1999) Segundo tratado sobre el gobierno civil. Un ensayo acerca del verdadero origen, alcance y fin del gobierno civil. Madrid: Alianza.

- Macfarlane, A. (1993). La cultura del capitalismo. México: Fondo de Cultura Económica.

- Osborne, R. (2007). Civilización, una historia crítica del mundo occidental. Barcelona: Editorial Crítica.

- Patiño, C. (2005). El origen del poder en Occidente. Bogotá: Siglo del Hombre.

- $\quad$ Reich, R. (1993). El trabajo de las naciones hacia el capitalismo del siglo XXI. Buenos Aires: Javier Vergara.

- Spinoza, B. (1986). Tratado teológico - político. Madrid: Alianza Editorial.

- $\quad$ Toynbee A. (1955). El mundo y el occidente. Madrid: Aguilar.

- Wallerstein, I. (2005). Un mundo incierto. Buenos Aires: Libros del Zorzal.

- Waltz, K. (1988). Teoría de la Política Internacional. Buenos Aires: GEL.

- Weber, M. (2003) La ética protestante y el espíritu del capitalismo. Madrid: Alianza Editorial.

- Wilhelmy von Wolff, M. (1988). Política internacional: enfoques y realidades. Buenos Aires: Grupo Editor Latinoamericano. 